\title{
Tissue Changes in Experimental Cryptococcosis in Immunocompetent and Immunodeficient Murine Model
}

Eriques Gonçalves Silva ${ }^{1 *}$, Josemar Neves Cavalcanti ${ }^{2}$, Flavio Cesar Viani ${ }^{3}$, Sandra Marilia de Souza Silva ${ }^{4}$ and Amanda Latercia Tranches Dias ${ }^{5}$

${ }^{1}$ Governo do Estado de São Paulo-Secretaria de Estado da Educação, São Paulo, Brazil

${ }^{2}$ Faculdade de Americana - (FAM), Americana - São Paulo, Brazil

${ }^{3}$ Universidade Cruzeiro do Sul (UNICSUL) - São Paulo, Brazil

${ }^{4}$ Universidade Federal do ABC (UNIABC), Santo André, São Paulo, Brazil

${ }^{5}$ Universidade Federal de Alfenas (UNIFAL), Alfenas - Minas Gerais, Brazil

\begin{abstract}
Cryptococcosis is a subacute or chronic disease caused through the inhalation of infectious particles from the opportunistic yeast Cryptococcus neoformans spp. The objective of the present study was to evaluate cryptococcosis in a murine model Immunocompetent (BALB/c), as well as in a model with combined immunodeficiency (SCID) through histopathological analyzes of pulmonary and cerebral tissues. After intravenous inoculation with $3.0 \times 10^{5}$ viable yeast cells the animals were euthanized daily for evaluation. The study period was 15 days. There were no significant changes in lung tissue in immunocompetent murine model (BALB/C). While in brain tissue, it was observed: congested vessel, evolving when $C$. neoformans was visualized in the meningeal area, and a large area of ischemia, which evolved throughout the studied period culminating on the $15^{\text {th }}$ day of inoculation with visualization of the yeast in the meningeal and parenchyma. In SCID model, twenty-four hours after inoculation were observed in the lung tissue, hemorrhagic areas and a discrete neutrophilic inflammatory infiltrate, presence of discrete congestion in the lung, diffuse hemorrhage, edema and intense quantity of yeast were observed on the wall of the capillary at 11 days after inoculation. In brain tissue discrete area necrosis liquefaction was observed, focal well as the presence of $C$. neoformans, interspersed with fragments of necrotic cells was observed. On day 11 after inoculation were large areas of liquefaction necrosis associated with the formation of cavities in the parenchyma and an intense quantify of the yeast. Histopathological examination is one of the techniques usually used in the definitive diagnosis of cryptococcosis.
\end{abstract}

Keywords: Chronic disease; Cryptococcosis; C. neoformans; Necrosis

\section{Introduction}

Cryptococcosis diseases are caused through the inhalation of infective particles of the opportunistic C. neoformans, which stay in the lungs, causing pulmonary cryptococcosis or through the bloodstream, the yeast can arrives at other organs, mainly in the central nervous system (CNS), determinant of mortality in mice in experimental systemic infection. In the brain, C. neoformans can infect the meninges and through the cerebrospinal fluid (CSF), and the entire subarachnoid space (meningitis), or present as deep abscess and bulky (crytococcoma), which is its most common in immunocompetent patients [1]. The Crytococcoma is characterized as a solid tumor that occurs due to a granulomatous reaction composed of macrophages and lymphocytes, not common in immunosuppressed patients due to the inability of these patients to initiate an inflammatory response [2]. Most studies in experimental Cryptococcosis have been performed using murine model studies using these models are well established in many areas of research, including fungal pathogenesis $[3,4]$. The cerebral cryptococcosis in a murine model accurately reproduces the main features of the disease in humans and can be used to study various aspects of the pathophysiology of cryptococcal meningitis [5], being a valuable tool that contributes to the understanding of how these infections occur [6]. The model of systemic cryptococcosis in mice with severe combined immunodeficiency (SCID) is useful for immunological and therapeutic study of the disease in immunodeficient hosts [7].

The objective of the study is to evaluate tissue changes in experimental cryptococcosis in immunocompetent and immunodeficient murine model.

\section{Materials and Methods}

\section{Cryptococcus neoformans}

The study was performed using the C. neoformans ATCC 90112 (serotype A). This strain was maintained in tubes containing Sabouraud dextrose agar (Difco Laboratories, Detroit, MI, USA) and glycerol at $-20^{\circ} \mathrm{C}$, in the Laboratory of Pathogenic Yeasts of the Department of Microbiology, at the Institute of Biomedical Sciences at São Paulo University, São Paulo, Brazil.

\section{Preparation of the inoculum}

The C. neoformans ATCC 90112 was cultivated in a YPD medium of $1 \%$ yeast extract (Difco), $1 \%$ Bacto Peptone (Difco), and $2 \%$ dextrose (Sigma-Aldrich, Milwaukee, WI, USA) for $18 \mathrm{~h}$ at $30^{\circ} \mathrm{C}$; the cells were collected after centrifugation, washed twice in a phosphate buffer solution (PBS), and resuspended at the inoculation concentration.

\section{Animal models and experimental cryptococcosis}

A total of 55 male BALB/c mice, weighing 24-27 g and 55 murine models with Severe Combinated Immunodeficiency (SCID), with and a mean weight of $20 \mathrm{~g}$, were used in the study. Were obtained from the Animal Center, which is responsible for breeding isogenic animals at the Institute for Energy and Nuclear Research, in São Paulo, Brazil.

*Corresponding author: Eriques Gonçalves Silva, Governo do Estado de São Paulo-Secretaria de Estado da Educação, São Paulo, Brazil, Tel: 5511970512752; E-mail: eriques@usp.br

Received January 15, 2018; Accepted January 25, 2018; Published January 29 2018

Citation: Silva EG, Cavalcanti JN, Viani FC, Silva SMD, Dias ALT (2018) Tissue Changes in Experimental Cryptococcosis in Immunocompetent and Immunodeficient Murine Model. J Med Microb Diagn 7: 271. doi:10.4172/2161. 0703.1000271

Copyright: ( 2018 Silva EG, et al. This is an open-access article distributed under the terms of the Creative Commons Attribution License, which permits unrestricted use, distribution, and reproduction in any medium, provided the original author and source are credited. 
These mice were housed in microisolator cages, provided with sterile feed and water. The animal's immunocompetent mice (BALB/c) and the immunodeficient (SCID) mice were randomly distributed into 10 groups of 5 animals in each group and were inoculated intravenously with $100 \mu \mathrm{L}$ of the suspension, containing $3.0 \times 10^{5}$ viable cells of the yeast. Nine groups were euthanized in the various moments $(1,2,3,5,7$, $9,11,13$ and 15 days) after infection to be evaluated; one group was not euthanized, serving as the positive control; and one group $(n=5)$ was inoculated with PBS, serving as the negative control, were maintained during the study period (50 days). This experiment was conducted in accordance with the Ethical Principles in Animal Research adopted by the Brazilian College of Animal Experimentation and was approved by the Ethical Committee for Animal Research of the Biomedical Sciences Institute/USP.

\section{Histopathological analysis}

At the end of each moment the study, all mice were euthanized in a $\mathrm{CO}_{2}$ chamber and were evaluated. Fragments the brains and lungs were aseptically removed washed in $50 \mathrm{mM}$ phosphate buffer, $\mathrm{pH}$ 7.4, dehydrated in graded concentrations of ethanol and then embedded in paraffin. From each brain and lung, $4 \mu \mathrm{m}$ sections were obtained and stained with hematoxylin and eosin (HE) and PAS (Acid Periodic de Schiff) to histopathological analysis. The slides were systematically scanned in an Axiophot Zeiss microscope with digital camera Canon G5.

\section{Results}

\section{Lung}

In immunodeficient murine model (BALB/c-SCID), twenty-four hours after inoculation were observed in the lung tissue, hemorrhagic areas and a discrete neutrophilic inflammatory infiltrate without observation of C. neoformans, (Figure 1). On day 5 was observed in the presence of discrete pulmonary congestion, (Figure 1). Diffuse hemorrhage, edema and intense quantity of yeast were observed on the wall of the capillary at 11 days after inoculation, (Figure 2). There were no significant changes in lung tissue in immunocompetent murine model $(\mathrm{BALB} / \mathrm{c})$.

\section{Brain}

Discrete area liquefaction necrosis, focal well as the presence of $C$. neoformans, interspersed with fragments of necrotic cells was observed on the $5^{\text {th }}$ and $7^{\text {th }}$ days after inoculation (Figure 3). On day 11 after inoculation were seen large areas of liquefaction necrosis associated with the formation of cavities in the parenchyma, containing inside fragments of necrotic cells and an intense quantify of C. neoformans (Figure 4), in immunocompetent murine model (BALB/c-SCID).

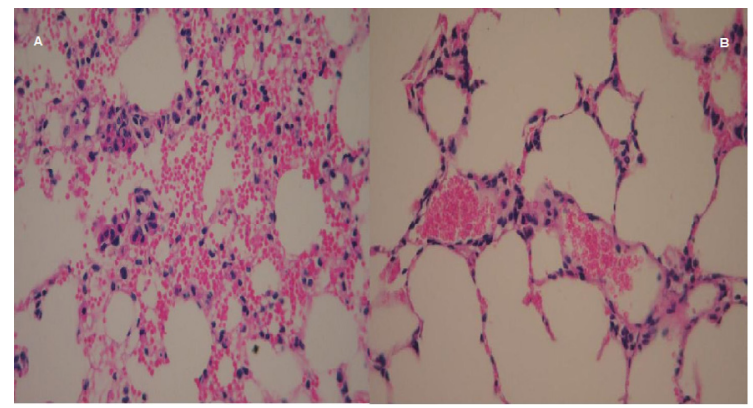

Figure 1: Histopathology of lung tissue in immunodeficient murine model. A, 1 day after inoculation showing focal areas of hemorrhage and discreet neutrophilic inflammatory infiltrate in the alveolar wall, $B, 5$ days after inoculation with mild pulmonary congestion, $\mathrm{HE}, 400 \mathrm{X}$

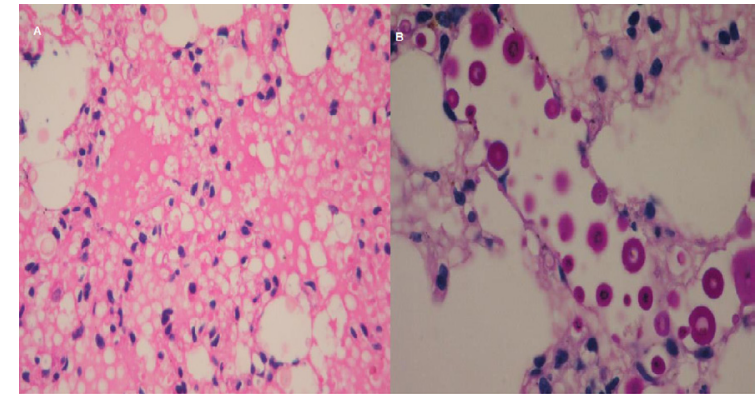

Figure 2: Histopathology of lung tissue in immunodeficient murine model 11 days after inoculation. A - presenting edema, congestion, hemorrhage, diffuse and focal neutrophilic infiltrate, $\mathrm{HE}, 400 \mathrm{x}, \mathrm{B}$ - Intense quantify of $C$. neoformans Observed in the capillaries of the alveolar wall, PAS, 1000.

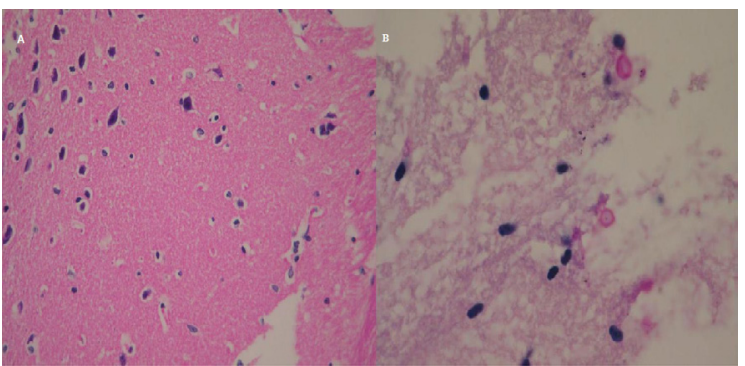

Figure 3: Histopathology of brain tissue in immunodeficient murine model, 5 days after inoculation, A - presenting a discrete area of liquefaction necrosis, focal HE, 400X, B - C. neoformans displayed, interspersed with fragments of necrotic cells, 7 days after inoculation, PAS, 400X.

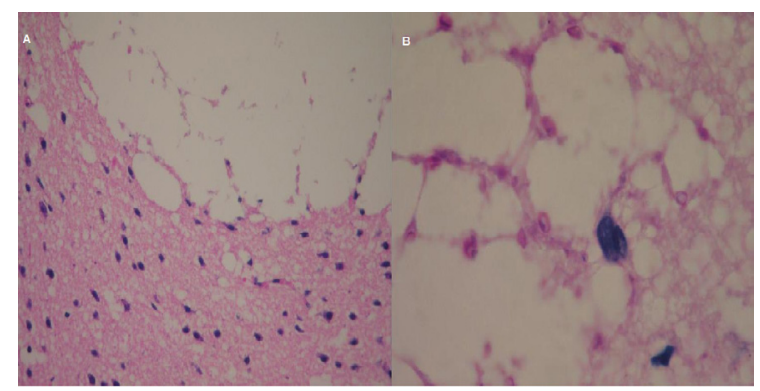

Figure 4: Histopathology of brain tissue in immunodeficient murine model at 11 days after the inoculation; $A$ - presenting large areas of necrosis associated with liquefaction of cavitations in the parenchyma, HE, 400X; B - quantify of intense C. neoformans displayed inside the cavities, PAS, 400X.

In an immunocompetent murine model $(\mathrm{BALB} / \mathrm{c})$ were observed edema and congested vessels, 24 and 48 hours, respectively, after inoculation (Figure 5). C. neoformans was observed in the meningeal with 7 days of inoculation, as well as a mononuclear inflammatory infiltrate at day 9, (Figure 6). Were observed, a large ischemic area, and crytococcoma, at $11^{\text {th }}$ and $13^{\text {th }}$ days after inoculation, respectively, (Figure 7). In the (Figure 8), there is C. neoformans in the meninges and parenchyma 15 days after inoculation.

\section{Discussion}

A murine model with Severe Combined Immunodeficiency (BALB/c-SCID), resulting from a recessive mutation that occurs on chromosome 16, this mutation causes these animals to exhibit a deficiency of $\mathrm{T}$ and $\mathrm{B}$ cell-mediated immunity, making them more susceptible to systemic experimental cryptococcosis $[7,8]$. The present 


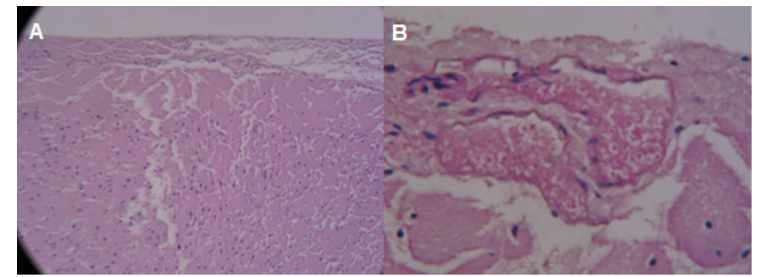

Figure 5: Histopathology of brain tissue in immunocompetent murine model. The - 24 hours after inoculation presenting edema, HE, 200X, B - 48 hours after inoculation vessel congested, HE, 200X

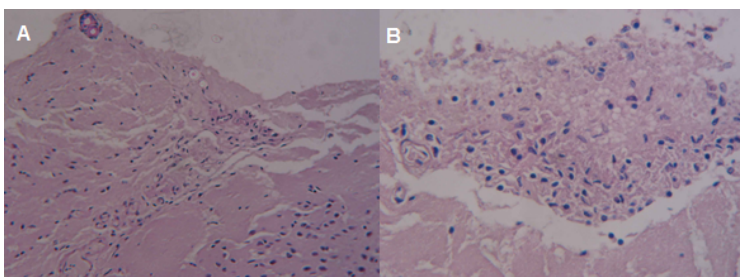

Figure 6: Histopathology of brain tissue in immunocompetent murine model. A - C. neoformans observed on meninges, 7 days after inoculation, HE, 200X, B - mononuclear inflammatory infiltrate in the 9th day after inoculation, $\mathrm{HE}, 500 \mathrm{X}$.

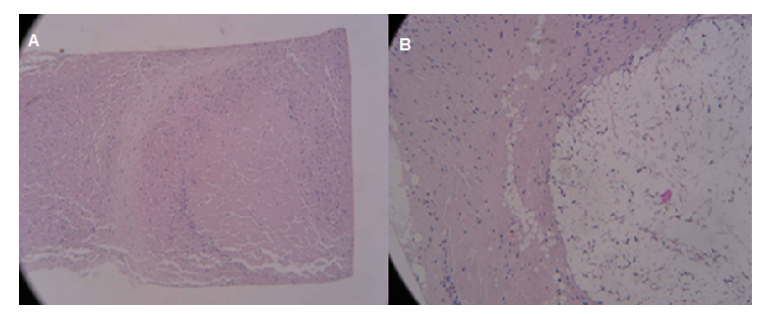

Figure 7: Histopathology of brain tissue in immunocompetent murine model. A - Large ischemic area 11 days after inoculation, HE, 100X, B Crytococcoma giant 13 days after inoculation, HE, 200X.

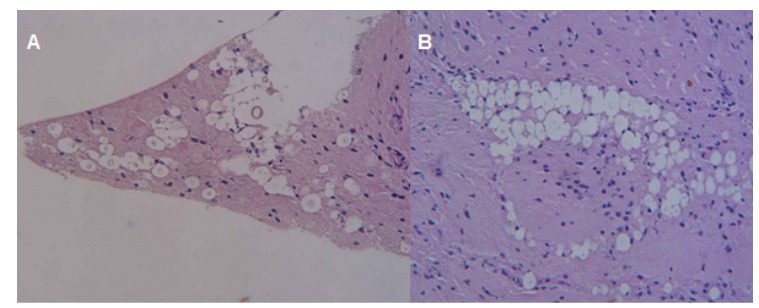

Figure 8: Histopathology of brain tissue in immunocompetent murine model, 15 days after inoculation. A - C. neoformans in the meninges, HE, 200X, B - C. neoformans in the parenchyma, HE, 500X.

study evaluated the histopathological changes in experimental cryptococcosis in an immunocompetent animal model (BALB/c), as well as in the murine immunodeficient model (BALB/c-SCID) through tissue changes in the lungs, as well as in the brains of these animals. Histopathological examination is one of the techniques usually used in the definitive diagnosis of cryptococcosis [9].

Under the conditions studied, there were no significant tissue changes in immunocompetent murine lung tissue. While in an immunodeficient model with 24 hours it was possible to verify bleeding in a focal pattern and a discrete neutrophilic infiltrate. Although we verified in our study the presence of a neutrophilic inflammatory infiltrate in the alveolar wall $24 \mathrm{~h}$ after infection, we found that it was not able to resolve the infection. Clinical manifestations of pulmonary cryptococcosis include localized nodular lesions, with or without cavitation, segmental pneumococcal infiltrates, interstitial or alveolar infiltrate, pleural effusion, hilar masses, and thoracic lymphadenopathy. Migration of neutrophils into lung tissue is important in the early protection of mice against progressive cryptococcosis [10-15].

The host defense efficacy against C. neoformans depends on a combination of several mechanisms, such as natural and immune [15], in humans, T CD4 cell deficiency is associated with disseminated cryptococcosis [16]. The humoral response, especially immunoglobulin G, also enhances host defense against cryptococcosis [17], however, T cells have a modulatory effect on $\mathrm{B}$ cell response against $C$. neoformans infection in mice [18].

In the murine immunodeficient model (BALB / c-SCID) hemorrhagic areas were observed in pulmonary tissue, as well as a discrete neutrophilic infiltrate twenty-four hours after inoculation, culminating with pulmonary congestion on day 5 . The course of lung infection is characterized by a rapid response of neutrophils, with cryptococcus phagocytosis [19], being that the phagocytosis and the death of $C$. neoformans by neutrophils is diminished due to the presence of the very large polysaccharide capsule [20].

In the model studied, a large amount of yeast in the wall of the capillary vessels was observed around the 11th day of inoculation, it can be inferred that the pulmonary cryptococcosis occurred during this period, culminating with the death of the animals, during that period a cryptococcosis where the yeast present in the alveolar wall and the presence of edema were found. As previously reported, cryptococcosis occurs primarily in the lungs [21].

In immunocompromised patients, pulmonary lesion initially consists of intraalveolar proliferation of $C$. neoformans with a response to histiocytes. The yeast is prominently visualized in the capillaries. In a second moment, it is possible to observe that $C$. neoformans is widely distributed in the tissue, involving innumerable alveoli, and finally a massive proliferation of the yeast occurs in both alveoli and interstitium, causing the destruction of the capillaries, resulting in a focal hemorrhage at this moment.

In the brain tissue through the histopathological analyzes we can highlight the presence of a discrete area of focal liquefaction necrosis on the $5^{\text {th }}$ day after the initial infection. In this period we also observed C. neoformans interspersed with fragments of necrotic cells, being most often visualized in the lumen of the blood vessels. In cryptococcal meningitis local inflammation is limited [22] and lesions are described as dilatation of the perivascular spaces as well as, brain mass [5]. In the present study no inflammatory reaction was observed in this tissue. Metabolites produced by $C$. neoformans can cause necrosis and death of neutrophils [23]. We did not observe C. neoformans associated with the meningeal.

Chang et al. [3], reported in their work that the yeast was found associated with the meningeum 10 days after the initial infection. It is believed that $C$. neoformans reaches the brain through capillaries $[3,4]$.

On the 11th day of infection, we observed extensive areas of necrosis of liquefaction in the brain, associated with the formation of cavitations in the parenchyma, where it was possible to visualize that inside these cavitations fragments of necrotic cells and an intense amount of C. neoformans. Contrary to what was seen in immunocompetent models, in the studied SCID model we did not observe the presence of cryptococoma, nor any inflammatory response. Although the 
Citation: Silva EG, Cavalcanti JN, Viani FC, Silva SMD, Dias ALT (2018) Tissue Changes in Experimental Cryptococcosis in Immunocompetent and Immunodeficient Murine Model. J Med Microb Diagn 7: 271. doi:10.4172/2161-0703.1000271

experimental infection in this study was absent through intravenous inoculation of the yeast, we can also suggest that the entrance port of C. neoformans in the lung, in this model studied are the pulmonary alveoli, a fact confirmed when visualizing the yeast in the most of the time adhered to the cell wall of the alveoli. The lung is considered to be the gateway to infection [24]. In natural infection, yeast spores can be spread through the air and infect susceptible humans, the portal being the airways, lodged primarily in the pulmonary alveoli [25]. Our data also lead us to infer that the death of the animals was due to lung infection and not to meningoencephalitis, because in the histopathological study of the brain tissue of the animals studied it was not possible to visualize $C$. neoformans associated to the meninges as already described by other authors $[3,4]$.

\section{Conclusion}

We can suggest that yeast access to the brain is via the bloodstream and histopathological examination is one of the techniques usually used in the definitive diagnosis of cryptococcosis.

\section{Funding}

The authors are grateful to FAPESP and CNPq for financial support.

\section{Conflict of Interest Statement}

There is no conflict of interest in the present study.

\section{References}

1. Kesler R, Maertens $P$ (1999) Pontine cryptococcoma in non-immunocompromised individual: MRI characteristics. J Neuroimaging 9: 118-121.

2. Rambeloarisoa J, Batisse D, Thiebaut JB, Mikol J, Mrejen S, et al. (2002) Intramedullary abscess resulting from disseminated cryptococcosis despite immune restoration in a patient with AIDS. J Infect 44: 185-188.

3. Chang YC, Stins MF, McCaffery MJ, Miller GF, Pare DR, et al. (2004) Cryptococcal yeast cells invade the central nervous system via transcellular penetration of the blood-brain barrier. Infect Immun 72: 4985-1995.

4. Charlier C, Chrétien F, Baudrimont M, Mordelet E, Lortholary O, et al. (2005) Capsule structure changes associated with Cryptococcus neoformans crossing of the blood-brain barrier. Am J Pathol 166: 421-432.

5. Chrétien F, Lortholary O, Kansau I, Neuville S, Gray F, et al. (2002) Pathogenesis of cerebral Cryptococcus neoformans infection after fungemia. J Infect Dis 186: 522-530.

6. Capilla J, Clemons K, Stevens D (2007) Animal models: An important tool in mycology. Med Mycol 45: 657-684.

7. Clemons K, Azzi R, Stevens D (1996) Experimental systemic cryptococcosis in SCID mice. J Med Vet Mycol 34: 331-335.

8. Huffnagle GB, Yates JL, Lipscomb MF (1991) T cell-mediated immunity in the lung: a Cryptococcus neoformans pulmonary infection model using SCID and athymic nude mice. Infect Immun 59: 1423-1433.
9. Xavier MO, Oliveira FEM, Severo LC (2009) Chapter 1: Laboratory diagnosis of pulmonary mycoses. J Bras Pneumol 35: 907-919.

10. Guillot L, Carroll SF, Homer R, Qureshi ST (2008) Enhanced innate immune responsiveness to pulmonary Cryptococcus neoformans infection is associated with resistance to progressive infection. Infect Immun 76: 4745-4756.

11. Ellerbroek P, Lefeber DJ, Van Veghel R, Scharringa J, Brouwer E, et al. (2004) O-acetylation of cryptococcal capsular glucuronoxylomannan is essential for interference with neutrophil migration. J Immunol 173: 7513-7520.

12. Guy JP, Raza S, Bondi E, Rosen Y, Kim DS, et al. (2012) Cryptococcus pneumonia presenting in an immunocompetent host with pulmonary asbestosis: a case report. J Med Case Rep 6: 170.

13. Wozniak K, Ravi S, Macias S, Young ML, Olszewski MA, et al. (2009) Insights into the mechanisms of protective immunity against Cryptococcus neoformans infection using a mouse model of pulmonary cryptococcosis. PLoS One 4: e6854.

14. Zhang Y, Wang F, Tompkins KC, McNamara A, Jain AV, et al. (2009) Robust Th1 and Th17 immunity supports pulmonary clearance but cannot prevent systemic dissemination of highly virulent Cryptococcus neoformans H99. Am J Pathol 175: 2489-2500.

15. Murphy J (1991) Mechanisms of natural resistance to human pathogenic fungi. Annu Rev Microbiol 45: 509-538.

16. Duncan R, Von Reyn CF, Alliegro GM, Toossi Z, Sugar AM, et al. (1993) Idiopathic CD4+ T-lymphocytopenia--four patients with opportunistic infections and no evidence of HIV infection. N Engl J Med 328: 393-398.

17. Rivera J, Zaragoza O, Casadevall A (2005) Antibody-mediated protection against Cryptococcus neoformans pulmonary infection is dependent on $B$ cells. Infect Immun 73: 1141-1150.

18. Beenhouwer D, Shapiro S, Feldmesser M, Casadevall A, Scharff MD (2001) Both Th1 and Th2 cytokines affect the ability of monoclonal antibodies to protect mice against Cryptococcus neoformans. Infect Immun 69: 6445-6455.

19. Dong Z, Murphy J (1995) Effects of the two varieties of Cryptococcus neoformans cells and culture filtrate antigens on neutrophil locomotion. Infect Immun 63: 2632-2644.

20. Kozel T (1996) Activation of the complement system by pathogenic fungi. Clin Microbiol Rev 9: 34-46.

21. Buchanan K, Murphy J (1998) What makes Cryptococcus neoformans a pathogen? Emerg Infect Dis 4: 71-83.

22. Lortholary O, Dromer F, Mathoulin-Pélissier S, Fitting C, Improvisi L, et al (2001) Immune mediators in cerebrospinal fluid during cryptococcosis are influenced by meningeal involvement and human immunodeficiency virus serostatus. J Infect Dis 183: 294-302.

23. Wright L, Bubb W, Davidson J, Santangelo R, Krockenberger M, et al. (2002) Metabolites released by Cryptococcus neoformans var. neoformans and var. gatti differentially affect human neutrophil function. Microbes Infect 4: 1427-1438.

24. Salyer W, Salyer D, Baker R (1974) Primary complex of Cryptococcus and pulmonary lymph nodes. J Infect Dis 130: 74-77.

25. Sukroongreung S, Kitiniyom K, Nilakul C, Tantimavanich S (1998) Pathogenicity of basidiospores of Filobasidiella neoformans var. neoformans. Med Mycol 36 419-424. 